\title{
Malignant Jugulotympanic Paraganglioma
}

National Cancer Institute

\section{Source}

National Cancer Institute. Malignant Jugulotympanic Paraganglioma. NCI Thesaurus.

Code C4623.

A jugulotympanic paragang lioma that metastasizes to other anatomic sites. 